\title{
Eigenfunction Statistics for Anderson Model with Hölder continuous single site Potential
}

\author{
Dhriti Ranjan Dolai and Anish Mallick \\ The Institute of Mathematical Sciences \\ Taramani, Chennai 600113 \\ dhriti@imsc.res.in, anishm@imsc.res.in
}

February 15, 2018

\begin{abstract}
We consider Random Schrödinger operators on $\ell^{2}\left(\mathbb{Z}^{d}\right)$ with $\alpha$-Hölder continuous $(0<\alpha \leq 1)$ single site distribution. In localized regime we study the distribution of eigenfunctions in space and energy simultaneously. In a certain scaling limit we prove limit points are Poisson.
\end{abstract}

AMS 2010 MSC: 35J10, 81Q10, 35P20

Keywords: Anderson Model, Hölder continuous measure, Poisson statistics.

\section{Introduction}

The Random Schrödinger operators $\left\{H^{\omega}\right\}_{\omega \in \Omega}$ on $\ell^{2}\left(\mathbb{Z}^{d}\right)$ is given by

$$
H^{\omega}=\Delta+V^{\omega}, \quad \omega \in \Omega
$$

where $\Delta$ is discrete Laplacian defined by

$$
(\Delta u)(n)=\sum_{|m-n|=1} u(m) \quad \forall n \in \mathbb{Z}^{d} u \in \ell^{2}\left(\mathbb{Z}^{d}\right)
$$

and random potential $V^{\omega}$ is defined by

$$
V^{\omega}=\sum_{n \in \mathbb{Z}^{d}} \omega_{n}\left|\delta_{n}\right\rangle\left\langle\delta_{n}\right| .
$$


where $\left\{\delta_{n}\right\}_{n \in \mathbb{Z}^{d}}$ is the standard basis for $\ell^{2}\left(\mathbb{Z}^{d}\right)$ and $\left\{\omega_{n}\right\}_{n \in \mathbb{Z}^{d}}$ are real valued iid random variables with common probability distribution $\mu$ with compact support. The probability space $\left(\mathbb{R}^{\mathbb{Z}^{d}}, \mathcal{B}_{\mathbb{R}^{d}}, \otimes_{\mathbb{Z}^{d}} \mu\right)$ is constructed via Kolmogorov theorem and will be denoted by $(\Omega, \mathcal{B}, \mathbb{P})$, and $\omega_{n}: \Omega \rightarrow \mathbb{R}$ are projection on $n^{\text {th }}$ coordinate.

For any bounded set $B \subset \mathbb{R}^{d}$ we consider the orthogonal projection $\chi_{B}$ onto $\ell^{2}\left(B \cap \mathbb{Z}^{d}\right)$ and define the matrices

$$
H_{B}^{\omega}=\left(\left\langle\delta_{n}, H^{\omega} \delta_{m}\right\rangle\right)_{n, m \in B}, G^{B}(z ; n, m)=\left\langle\delta_{n},\left(H_{B}^{\omega}-z\right)^{-1} \delta_{m}\right\rangle, G^{B}(z)=\left(H_{B}^{\omega}-z\right)^{-1} .
$$

Note that $H_{B}^{\omega}$ is the matrix

$$
\chi_{B} H^{\omega} \chi_{B}: \ell^{2}(B) \rightarrow \ell^{2}(B) \text { a.e } \omega .
$$

Let $E_{H_{B}^{\omega}}(\cdot)$ be the spectral projection of $H_{B}^{\omega}$.

Set the resolvent operator and it's matrix elements (Green's function) as:

$$
G(z)=\left(H^{\omega}-z\right)^{-1}, \quad G(z ; n, m)=\left\langle\delta_{n},\left(H^{\omega}-z\right)^{-1} \delta_{m}\right\rangle \quad z \in \mathbb{C}^{+} .
$$

Throughout this article we will be assuming following two conditions:

(a) The single site distribution $\mu$ is uniformly $\alpha$-Hölder continuous for some $0<\alpha \leq 1$.

(b) For any $0<s<1$ there exists $r, C>0$ such that for any $\Lambda \subseteq \mathbb{Z}^{d}$

$$
\sup _{\substack{z \in \mathbb{C}^{+} \\ \operatorname{Re}(z) \in[a, b]}} \mathbb{E}\left[\left|G^{\Lambda}(z ; n, m)\right|^{s}\right] \leq C e^{-r|n-m|}
$$

for any $n, m \in \Lambda$.

When the energy $E$ lies in $[a, b]$, then we say that $E$ is in localized regime. Using the resolvent identity we have

$$
\lim _{\Lambda \uparrow \mathbb{Z}^{d}} G^{\Lambda}(z ; n, m)=G(z ; n, m) \quad \text { a.e } \omega
$$

for $z \in \mathbb{C}^{+}$, so (1.4) holds for $\mathbb{E}\left[|G(z ; n, m)|^{s}\right]$ with same constant $C, r$. The condition (b) was established by Aizenman-Molchanov [2] at high disorder for $\alpha$-Hölder continuous single site distribution. Refer to [2, inequalities (2.10), (3.19) and (3.20)] for more details. 
It was shown by Krishna [22, Combes-Hislop-Klopp [6] and Combes-GerminetKlein [7] that whenever the single site distribution is uniformly $\alpha$-Hölder continuous the Integrated density of states (IDS) is also uniformly $\alpha$-Hölder continuous, $0<\alpha \leq 1$.

Before describing our main result, we need some notations in place. Let $\nu$ be the Integrated density of states (IDS) for the operator $H^{\omega}$. Define the fractional derivatives:

$$
d_{\nu}^{\alpha}(x)=\lim _{\epsilon \rightarrow 0} \frac{\nu(x-\epsilon, x+\epsilon)}{(2 \epsilon)^{\alpha}} \text { and } D_{\nu}^{\alpha}(x)=\varlimsup_{\epsilon \rightarrow 0} \frac{\nu(x-\epsilon, x+\epsilon)}{(2 \epsilon)^{\alpha}}
$$

Let $g: \mathbb{R}^{d} \longrightarrow \mathbb{R}^{+}$and $f: \mathbb{R} \longrightarrow \mathbb{R}^{+}$be compactly supported continuous functions. For a self adjoint operator $H$ on $\ell^{2}\left(\mathbb{Z}^{d}\right)$ with pure point spectrum i.e $\sigma(H)=\sigma_{p p}(H)$, define $M_{g}$ as the multiplication operator by $g$ :

$$
\left(M_{g} u\right)=g(n) u(n) \quad \forall n \in \mathbb{Z}^{d}, u \in \ell^{2}\left(\mathbb{Z}^{d}\right) .
$$

Let $\left\{E_{j}\right\}_{j}$ be the eigenvalues (repeated according to multiplicity) of $H$ and $\psi_{j}$ be the normalized eigenfunction corresponding to eigenvalue $E_{j}$. Then

$$
\operatorname{Tr}\left(M_{g} f(H)\right)=\sum_{j} \sum_{n \in \mathbb{Z}^{d}} f\left(E_{j}\right) g(n)\left|\psi_{j}(n)\right|^{2} .
$$

Define the random measure $\xi^{\omega}$ on $\mathbb{R}^{1+d}$ by

$$
\int_{\mathbb{R} \times \mathbb{R}^{d}} f(E, x) d \xi^{\omega}(E, x)=\sum_{j} \sum_{n \in \mathbb{Z}^{d}} f\left(E_{j}, n\right)\left|\psi_{j}(n)\right|^{2} \quad \forall f \in C_{c}\left(\mathbb{R} \times \mathbb{R}^{d}\right),
$$

following the notations of (1.6). Following the notation from physics literature

$$
d \xi^{\omega}(E, x)=\sum_{j} \sum_{n}\left|\psi_{j}(n)\right|^{2} \delta\left(E-E_{j}\right) d E \delta(x-n) d x
$$

where $\delta(x)$ is the Dirac-delta distribution. So for any Borel set $I \in \mathcal{B}_{\mathbb{R}}$ and $Q \in \mathcal{B}_{\mathbb{R}^{d}}$ we have

$$
\xi^{\omega}(I \times Q)=\operatorname{Tr}\left(\chi_{Q} E_{H^{\omega}}(I) \chi_{Q}\right)
$$

Killip-Nakano [19] studied eigenfunction statistics for discrete Anderson Model with bounded density. There they studied the sequence of random measures given by

$$
\int f(E, x) d \theta_{L, \lambda}^{\omega}(E, x)=\int_{\mathbb{R} \times \mathbb{R}^{d}} f\left(L^{d}(E-\lambda), \frac{x}{L}\right) d \xi^{\omega}(E, x) \quad \forall f \in C_{c}\left(\mathbb{R} \times \mathbb{R}^{d}\right)
$$


and proved its convergence to Poisson point process. Nakano in [26] worked with Continuum Schrödinger operator and was able to show the infinite divisibility of the limiting process. We are interested in similar object, we will study the limit of the random measures $\xi_{L, \lambda}^{\omega}$ defined by

$$
\int_{\mathbb{R} \times \mathbb{R}^{d}} f(E, x) d \xi_{L, \lambda}^{\omega}(E, x):=\int_{\mathbb{R} \times \mathbb{R}^{d}} f\left(\beta_{L}(E-\lambda), \frac{x}{L}\right) d \xi^{\omega}(E, x) \quad \forall f \in C_{c}\left(\mathbb{R} \times \mathbb{R}^{d}\right),
$$

where $\lambda \in[a, b]$ satisfying (1.4); equivalently

$$
\xi_{L, \lambda}^{\omega}(I \times Q)=\operatorname{Tr}\left(\chi_{L Q} E_{H^{\omega}}\left(\lambda+\beta_{L}^{-1} I\right) \chi_{L Q}\right), \quad I \in \mathcal{B}_{\mathbb{R}}, Q \in \mathcal{B}_{\mathbb{R}^{d}}
$$

with $\beta_{L}=L^{d / \alpha}$. $\beta_{L}$ is chosen based on the work of Dolai-Krishna [11]. They used $\beta_{L}$ as scaling factor for eigenvalue statistics and showed the convergence to a Poisson random variable. Our main result is the following theorem.

Theorem 1.1. Let $\xi_{L, \lambda}^{\omega}$ be defined by (1.10) where $H^{\omega}$ is given by (1.1) and $\mu, \lambda$ follow assumptions (a) and (b). Let $I \subset \mathbb{R}$ be a bounded symmetric interval and $Q \subset \mathbb{R}^{d}$ be a rectangles with sides parallel to axes. Then there exists a subsequence $\left\{L_{n}\right\}$ such that the sequence of random variables $\left\{\xi_{L_{n}, \lambda}^{\omega}(I \times Q)\right\}$ converge in distribution to a Poisson random variable with parameter $|I|^{\alpha} D_{\nu}^{\alpha}(\lambda)|Q|=\gamma_{\lambda}(I \times Q)$, whenever $0<D_{\nu}^{\alpha}(\lambda)<\infty$.

Remark 1.2. For sequence $\left\{\xi_{L_{n}, \lambda}^{\omega}(I \times Q)\right\}_{n}$ to converge, the sequence $\left\{L_{n}\right\}_{n}$ depends only on $I$ and $\lambda$ but not on $Q$. This is because:

$$
\begin{aligned}
\gamma_{\lambda}(I \times Q) & =\lim _{n \rightarrow \infty} \mathbb{E}\left[\xi_{\lambda, L_{n}}^{\omega}(I \times Q)\right] \\
& =\lim _{n \rightarrow \infty} \mathbb{E}\left[\sum_{m \in L Q \cap \mathbb{Z}^{d}}\left\langle\delta_{m}, E_{H^{\omega}}\left(\lambda+\beta_{L}^{-1} I\right) \delta_{m}\right\rangle\right] \\
& =\lim _{n \rightarrow \infty}\left(|Q| L_{n}^{d}+o\left(L_{n}^{d-1}\right)\right) \mathbb{E}\left[\left\langle\delta_{0}, E_{H^{\omega}}\left(\lambda+\beta_{L_{n}}^{-1} I\right) \delta_{0}\right\rangle\right] \\
& =|Q| \lim _{n \rightarrow \infty} L_{n}^{d} \mathbb{E}\left[\left\langle\delta_{0}, E_{H^{\omega}}\left(\lambda+\beta_{L_{n}}^{-1} I\right) \delta_{0}\right\rangle\right] \\
& =|Q| \lim _{n \rightarrow \infty} L_{n}^{d} \nu\left(\lambda+\beta_{L_{n}}^{-1} I\right)=|Q||I|^{\alpha} D_{\nu}^{\alpha}(\lambda)
\end{aligned}
$$

the limit is obtained through lemma 2.6.

$D_{\nu}^{\alpha}$ is defined using symmetric intervals. In general, left and right $\alpha$-derivatives does not coincide with symmetric $\alpha$-derivative, while in case of usual derivative all three are same. It is also hard to determine the set $\left\{x: D_{\nu}^{\alpha}(x)>0\right\}$, 
so we have kept $D_{\nu}^{\alpha}(\lambda)>0$ in the hypothesis and considered the case of symmetric intervals only.

To compute the limit of $\xi_{L, \lambda}^{\omega}$ as a random measure over a subsequence $\left\{L_{n}\right\}_{n}$, we should be able to compute $\lim _{n \rightarrow \infty} \mathbb{E}_{\omega}\left[\xi_{L_{n}, \lambda}^{\omega}(I \times Q)\right]$ for any bounded interval I. Even if we consider $\xi_{L, \lambda}^{\omega}$ as random measure on the Borel $\sigma$-algebra $\mathscr{B}$ generated by $\{(-b, b) \backslash(-a, a): 0<a<b<\infty\}$, we have to take different subsequences for different $I \in \mathscr{B}$. On other hand if $d_{\nu}^{\alpha}(\lambda)$ exists, then

$$
\lim _{L \rightarrow \infty} \underset{\omega}{\mathbb{E}}\left[\xi_{L, \lambda}^{\omega}(I \times Q)\right]=\alpha 2^{\alpha-1} d_{\nu}^{\alpha}(\lambda)|Q| \int_{I} x^{\alpha-1} d x
$$

where $I$ is a generator of $\mathscr{B}$. In this case one can prove convergence as random measures. As a special case, we can consider $\left\{\xi_{L_{n}, \lambda}^{\omega}(I \times \cdot)\right\}_{n}$ as random measure for fixed interval, then

Corollary 1.3. For a fixed symmetric bounded interval $I \subset \mathbb{R}$, we consider the random measure $\left\{\xi_{L, \lambda}^{\omega}(I \times \cdot)\right\}$ on $\mathbb{R}^{d}$. There exists a subsequence $\left\{L_{n}\right\}$ such that $\left\{\xi_{L_{n}, \lambda}^{\omega}(I \times \cdot)\right\}$ converges weakly to a Poisson point process with intensity measure $|I|^{\alpha} D_{\nu}^{\alpha}(\lambda) d x$, where $d x$ is the Lebesgue measure on $\mathbb{R}^{d}$.

Using (iv) of [17, Theorem 16.16], the above Corollary is immediate once we have Theorem 1.1.

Eigenvalue statistics for one dimension was studied by Molchanov [24], and later for higher dimension by Minami [23]. In region of fractional localization (where (1.4) holds), they showed that the statistics is Poisson. Subsequently the Poisson statistics was shown for the trees by Aizenman-Warzel in [3] and recently Poisson statistics was obtained by Geisinger [12] for regular graphs. In recent results Germinet-Klopp [13] extended the results of [19].

Recently Kotani-Nakano [21] investigated the statistics for one dimensional decaying random Schrödinger operators on $L^{2}(\mathbb{R})$. An analogue of Minami's [23] work was done by Dolai-Krishna [11] with $\alpha$-Hölder continuous single site distribution. In [10] Dolai-Krishna considered the Anderson Model with decaying Random Potentials and showed that the statistics inside $[-2 d, 2 d]$ in dimension $d \geq 3$ is independent of the randomness and agrees with that of the free part $\Delta$. 


\section{Preliminaries}

Given $L$ large enough, define $l_{L}$ such that $l_{L} \approx L^{a}$ for some $0<a<1$. Define the boxes

$$
B_{p}(L)=\left\{x \in \mathbb{Z}^{d}: p_{j} l_{L} \leq x_{j}<\left(p_{j}+1\right) l_{L}, \text { for } i=1,2, \cdots d\right\}, \quad p \in \mathbb{Z}^{d} .
$$

Let $H_{B_{p}(L)}^{\omega}$ denote the restriction of $H^{\omega}$ to $B_{p}(L)$. For $\lambda$ in localized regime, define the random measure $\eta_{p, \lambda}^{\omega}$ associated with $H_{B_{p}(L)}^{\omega}$ by:

$$
\int_{\mathbb{R} \times \mathbb{R}^{d}} f(E, x) d \eta_{p, \lambda}^{\omega}(E, x)=\sum_{j} \sum_{n \in B_{p}(L)} f\left(\beta_{L}\left(E_{j}-\lambda\right), \frac{n}{L}\right)\left|\psi_{j}(n)\right|^{2}, f \in C_{c}\left(\mathbb{R} \times \mathbb{R}^{d}\right),
$$

where $\left\{E_{j}\right\}_{j}$ are the eigenvalues of $H_{B_{p}(L)}^{\omega}$ and $\psi_{j}$ are corresponding eigenfunctions. Equivalently

$$
\eta_{p, \lambda}^{\omega}(I \times Q)=\operatorname{Tr}\left(\chi_{L Q} E_{H_{B_{p}(L)}^{\omega}}\left(\lambda+\beta_{L}^{-1} I\right) \chi_{L Q}\right), \quad I \in \mathcal{B}_{\mathbb{R}}, Q \in \mathcal{B}_{\mathbb{R}^{d}} .
$$

Since $H_{B_{p}(L)}^{\omega}$ is a matrix, for $|I|<\infty$ and $|Q|<\infty$ we have,

$$
\eta_{p, \lambda}^{\omega}(I \times Q)<\infty .
$$

But it should be noted that it is not a point process.

Related to $B_{p}(L)$ we will need:

$$
\begin{gathered}
\partial B_{p}(L)=\left\{x \in B_{p}(L): \exists x^{\prime} \in \mathbb{Z}^{d} \backslash B_{p}(L) \text { such that }\left|x-x^{\prime}\right|=1\right\} \\
\operatorname{int}\left(B_{p}(L)\right)=\left\{x \in B_{p}(L): \operatorname{dist}\left(x, \partial B_{p}(L)\right)>N_{L}\right\}
\end{gathered}
$$

where $\left\{N_{L}\right\}_{L}$ is a increasing sequences of positive integer such that $N_{L} \approx$ $\gamma \ln L$, we will specify $\gamma$ later. Observe

$$
\left|B_{p}(L) \backslash \operatorname{int}\left(B_{p}(L)\right)\right|=O\left(l_{L}^{d-1} \ln L\right), \quad N_{L} \approx \gamma \ln L .
$$

Let $C_{p}(L)$ be the cube in $\mathbb{R}^{d}$ corresponding to $B_{p}(L)$ defined by

$$
C_{p}(L)=\left\{x \in \mathbb{R}^{d}: p_{j} l_{L} \leq x_{j}<\left(p_{j}+1\right) l_{L}, \text { for } i=1,2, \cdots d\right\}, \quad p \in \mathbb{Z}^{d} .
$$

So $B_{p}(L)=C_{p}(L) \cap \mathbb{Z}^{d}$.

Observe that $\mathbb{Z}^{d}$ (resp $\mathbb{R}^{d}$ ) can be expressed as disjoint union of $B_{p}(L)$ (respectively $\left.C_{p}(L)\right)$. 
For a Borel set $Q$ of finite diameter (i.e $\sup \{|x-y|, x, y \in Q\}<\infty$ ), there exists a finite finite set $\Gamma$ such that $Q \subseteq \cup_{\Gamma \in p} C_{p}(L)$. Let $\Gamma_{L} \subset \mathbb{Z}^{d}$ be such that

$$
L Q=\bigcup_{p \in \Gamma_{L}}\left(C_{p}(L) \cap L Q\right)
$$

Then $\left\{\eta_{p, \lambda}^{\omega}\right\}_{p \in \Gamma_{L}}$ are statistically independent. Also

$$
\left|\Gamma_{L}\right| \leq\left(\frac{L}{l_{L}}\right)^{d}|Q| .
$$

In the following whenever we write sum over $p$, we mean the sum is taken over $\Gamma_{L}$.

We will need Wegner and Minami type estimates given in Combes-GerminetKlein [7]. Hence following there notations, set $S_{\mu}(s)=\sup _{a \in \mathbb{R}} \mu[a, a+s]$ for probability measure $\mu$ and define

$$
Q_{\mu}(s)=\left\{\begin{aligned}
\|\rho\|_{\infty} s & \text { if } \mu \text { has bounded density } \\
8 S_{\mu}(s) & \text { otherwise. }
\end{aligned}\right.
$$

If $\mu$ is uniformly $\alpha$-Hölder continuous with $0<\alpha \leq 1$, then $S_{\mu}(s) \leq U s^{\alpha}$ for small $s>0$ for some constant $U$. Following estimates will be used:

Lemma 2.1. For all bounded interval $I \subset \mathbb{R}$ and any finite volume $\Lambda \subset \mathbb{Z}^{d}$, we have

$$
\begin{gathered}
\mathbb{E}\left(\left\langle\delta_{n}, E_{H^{\omega}}(I) \delta_{n}\right\rangle\right) \leq Q_{\mu}(|I|) \forall n \in \mathbb{Z}^{d}, \\
\mathbb{E}\left(\operatorname{Tr}\left(E_{H_{\Lambda}^{\omega}}(I)\right)\right) \leq Q_{\mu}(|I|)|\Lambda|, \\
\mathbb{E}\left(\operatorname{Tr}\left(E_{H_{\Lambda}^{\omega}}(I)\right)\left(\operatorname{Tr}\left(E_{H_{\Lambda}^{\omega}}(I)\right)-1\right)\right) \leq\left(Q_{\mu}(|I|)|\Lambda|\right)^{2} .
\end{gathered}
$$

Proof can be found in Combes-Germinet-Klein [7, inequality (2.2)] for (2.8), [7. Theorem 2.3] for inequality (2.9) and [7, Theorem 2.1] for the inequality (2.10).

The following Corollary is immediate from the above lemma.

Corollary 2.2. Consider $\nu$ the IDS of the operators $H^{\omega}$ satisfying the condition $(\mathbf{a})$. Then for any $\psi \in C_{c}(\mathbb{R})$ and $n \in \mathbb{Z}^{d}$, we have

$$
\begin{gathered}
\int_{\mathbb{R}} \psi(x) d \nu(x)=\mathbb{E}\left(\left\langle\delta_{n}, \psi\left(H^{\omega}\right) \delta_{n}\right\rangle\right) \leq\|\psi\|_{\infty} Q_{\mu}\left(\left|s_{\psi}\right|\right), s_{\psi}=\operatorname{supp} \psi . \\
\mathbb{E}\left(\operatorname{Tr}\left(\psi\left(H_{\Lambda}^{\omega}\right)\right)\right) \leq\|\psi\|_{\infty} Q_{\mu}\left(\left|s_{\psi}\right|\right)|\Lambda|
\end{gathered}
$$


Proposition 2.3. For any $f \in C_{c}\left(\mathbb{R} \times \mathbb{R}^{d}\right)$ we have,

$\mathbb{E}^{\omega}\left\{\left|\int f(E, x) d \xi_{L, \lambda}^{\omega}(E, x)-\sum_{p} \int f(E, x) d \eta_{p, \lambda}^{\omega}(E, x)\right|\right\} \rightarrow 0$ as $L \rightarrow \infty$.

Proof. Take $f(E, x)=h(E) g(x)$ where $g$ is continuous function with compact support on $\mathbb{R}^{d}$ and $h$ is of the form

$$
h(E)=\operatorname{Im} \frac{1}{E-z}, \operatorname{Im} z>0 .
$$

Since linear combination of functions of form $f$ are dense in $C_{c}\left(\mathbb{R} \times \mathbb{R}^{d}\right)$, to prove (2.13) it is sufficient to prove for $f$, see [15, Appendix: The StoneWeierstrass Gavotte] for details. Let supp $g=Q \subset \mathbb{R}^{d}$ (because $g$ has compact support, we have $\sup \{|x-y|, x, y \in Q\}<\infty$ and $|Q|<\infty)$. We have

$$
\begin{aligned}
\int f(E, x) d \xi_{L, \lambda}^{\omega}(E, x) & =\sum_{n} g_{L}(n)\left\langle\delta_{n}, h_{L}\left(H^{\omega}\right) \delta_{n}\right\rangle \\
& =\frac{1}{\beta_{L}} \sum_{n} g_{L}(n) \operatorname{Im} G\left(z_{L} ; n, n\right) .
\end{aligned}
$$

and

$$
\begin{aligned}
\sum_{p} \int f(E, x) d \eta_{p, \lambda}^{\omega}(E, x) & =\sum_{p} \sum_{n} g_{L}(n)\left\langle\delta_{n}, h_{L}\left(H^{\omega}\right) \delta_{n}\right\rangle \\
& =\frac{1}{\beta_{L}} \sum_{p} \sum_{n} g_{L}(n) \operatorname{Im} G^{B_{p}}\left(z_{L} ; n, n\right), \quad B_{p}=B_{p}(L)
\end{aligned}
$$

where $g_{L}(x)=g\left(\frac{x}{L}\right), z_{L}=\beta_{L}^{-1} z, \operatorname{Im} z>0$ and $h_{L}$ is given by

$$
h_{L}(E)=h\left(\beta_{L}(E-\lambda)\right)=\frac{1}{\beta_{L}} \operatorname{Im} \frac{1}{E-\lambda-\beta_{L}^{-1} z} .
$$

The support of $g_{L}$ is $L Q$, so from the inequalities (2.5) and (2.6) we see that the support of $g_{L}$ intersect only $O\left(\frac{L}{l_{L}}\right)^{d}\left(i . e\left|\Gamma_{L}\right|\right)$ many disjoint cubes $B_{p}(L)$. So from (2.15) and (2.16) we have

$$
\left|\int f d \xi_{L, \lambda}^{\omega}-\sum_{p} \int f d \eta_{p, \lambda}^{\omega}\right|=\frac{1}{\beta_{L}}\left|\sum_{n \in L Q} g_{L}(n) \operatorname{Im} G\left(z_{L} ; n, n\right)-\sum_{p \in \Gamma_{L}} \sum_{n \in B_{p}(L)} g_{L}(n) \operatorname{Im} G^{B_{p}}\left(z_{L} ; n, n\right)\right|
$$




$$
\leq \frac{\|g\|_{\infty}}{\beta_{L}} \sum_{p \in \Gamma_{L}} \sum_{n \in B_{p}(L)}\left|\operatorname{Im} G\left(z_{L} ; n, n\right)-\operatorname{Im} G^{B_{p}}\left(z_{L} ; n, n\right)\right| .
$$

For $n \in \operatorname{int}\left(B_{p}(L)\right)$ and $z \in \mathbb{C}^{+}$, we have the perturbation formula

$$
G\left(z_{L} ; n, n\right)-G^{B_{p}}\left(z_{L} ; n, n\right)=\sum_{(m, k) \in \partial B_{p}(L)} G\left(z_{L} ; n, k\right) G^{B_{p}}\left(z_{L} ; m, n\right)
$$

$(m, k) \in \partial B_{p}(L)$ means $m \in \partial B_{p}(L), k \in \mathbb{Z}^{d} \backslash B_{p}(L)$ such that $|m-k|=1$. Following steps from Minami [23], we use (2.18) in (2.17) and get

$$
\begin{aligned}
\left|\int f d \xi_{L, \lambda}^{\omega}-\sum_{p} \int f d \eta_{p, \lambda}^{\omega}\right| \leq & \frac{\|g\|_{\infty}}{\beta_{L}} \sum_{p \in \Gamma_{L}} \sum_{n \in B_{p} \backslash i n t\left(B_{p}\right)}\left[\operatorname{ImG}\left(z_{L} ; n, n\right)+\operatorname{Im}^{B_{p}}\left(z_{L} ; n, n\right)\right] \\
& \quad+\frac{\|g\|_{\infty}}{\beta_{L}} \sum_{p \in \Gamma_{L}} \sum_{n \in \operatorname{int}\left(B_{p}(L)\right)} \sum_{(m, k) \in \partial B_{p}(L)}\left|G\left(z_{L} ; n, k\right) G^{B_{p}}\left(z_{L} ; m, n\right)\right| \\
= & A_{L}+B_{L} .
\end{aligned}
$$

For $B_{L}$ we have

$$
\begin{aligned}
B_{L} & =\frac{\|g\|_{\infty}}{\beta_{L}} \sum_{p \in \Gamma_{L}} \sum_{n \in i n t\left(B_{p}(L)\right)} \sum_{(m, k) \in \partial B_{p}(L)}\left|G\left(z_{L} ; n, k\right) G^{B_{p}}\left(z_{L} ; m, n\right)\right| \\
& =\frac{\|g\|_{\infty}}{\beta_{L}} \sum_{p \in \Gamma_{L}} \sum_{n \in \operatorname{int}\left(B_{p}(L)\right)} \sum_{(m, k) \in \partial B_{p}(L)}\left|G\left(z_{L} ; n, k\right)\right|\left|G^{B_{p}}\left(z_{L} ; m, n\right)\right|^{s}\left|G^{B_{p}}\left(z_{L} ; m, n\right)\right|^{1-s} .
\end{aligned}
$$

Now $(m, k) \in \partial B_{p}(L)$ and $n \in \operatorname{int}\left(B_{p}(L)\right)$ so we have $|n-k|>N_{L}$, using the exponential decay of Green's function given in (1.4) we have

$$
\mathbb{E}^{\omega}\left(\left|G^{B_{p}}\left(z_{L} ; n, k\right)\right|^{s}\right) \leq C e^{-r N_{L}}
$$

we also have

$$
\left|G\left(z_{L} ; n, k\right)\right| \leq \frac{1}{\left|I m z_{L}\right|} \text { and }\left|G^{B_{p}}\left(z_{L} ; m, n\right)\right|^{1-s} \leq \frac{1}{\left|I m z_{L}\right|^{1-s}} .
$$

So using above together with (2.21) in (2.20) we get

$$
\mathbb{E}^{\omega}\left(B_{L}\right) \leq \frac{C\|g\|_{\infty}}{\beta_{L}\left|I m z_{L}\right|^{2-s}}\left|\Gamma_{L}\right| l_{L}^{d} l_{L}^{d-1} N_{L} e^{-r N_{L}} .
$$


We have $l_{L} \simeq L^{a}(0<a<1), \Gamma_{L}=O\left(\frac{L}{l_{L}}\right)^{d}, I m z_{L}=\beta_{L}^{-1} \tau, \tau>0$ taking $z=\sigma+i \tau$, and $\beta_{L}=L^{d / \alpha}$. Choose $\gamma$ so that

$$
\gamma>\frac{1}{r}\left[(1-s) \frac{d}{\alpha}+d+(d-1) a\right]
$$

in definition of $N_{L}$ in (2.4). Then from (2.22) we get

$$
\mathbb{E}^{\omega}\left(B_{L}\right)=O\left(\gamma L^{-\delta} \ln L\right), \delta=r \gamma-[(1-s) d / \alpha+d+(d-1) a]>0 .
$$

From Combes-Germinet-Klein [7, A.9] we have, for any $k>0$

$$
\operatorname{Im} z \mathbb{E}\left[\operatorname{Im} G^{\Lambda}(z ; n, n)\right] \leq \pi\left(1+\frac{k}{2}\right) S_{\mu}\left(\frac{2 \operatorname{Im} z}{k}\right) .
$$

Since $\operatorname{Im} z_{L}=\beta_{L}^{-1} \operatorname{Im} z$ with $\operatorname{Im} z>0$ so using $S_{\mu}(s) \leq U s^{\alpha}(\alpha$-Hölder continuity of $\mu$ ) we get

$$
\begin{aligned}
\frac{1}{\beta_{L}} \mathbb{E}\left[\operatorname{Im} G^{\Lambda}\left(z_{L} ; n, n\right)\right] & \leq \frac{1}{\operatorname{Imz}} \pi\left(1+\frac{k}{2}\right) S_{\mu}\left(\frac{2 \operatorname{Im} z_{L}}{k}\right), \Lambda=C_{p}, \Lambda_{L} \\
& \leq C\left(\frac{2 \beta_{L}^{-1} I m z}{k}\right)^{\alpha} \\
& \leq C L^{-d}, \quad\left(\text { since } \beta_{L}=L^{d / \alpha}\right) .
\end{aligned}
$$

From (2.19) and (2.4) we have

$$
\begin{aligned}
\mathbb{E}^{\omega}\left(A_{L}\right) & \leq 2 C \frac{\|g\|_{\infty}}{\beta_{L}}\left|\Gamma_{L}\right|\left|B_{p}(L) \backslash \operatorname{int} B_{p}(L)\right| N_{L} L^{-d} \\
& \approx C\left(\frac{L}{l_{L}}\right)^{d} l_{L}^{d-1} \gamma \ln L L^{-d} \\
& =O\left(L^{-a} \ln L\right), \quad l_{l}=L^{a}, 0<a<1 .
\end{aligned}
$$

Combining (2.23) and (2.26) gives

$$
\mathbb{E}^{\omega}\left(A_{L}\right)+\mathbb{E}^{\omega}\left(B_{L}\right) \stackrel{L \rightarrow \infty}{\longrightarrow} 0
$$

The above convergence together with (2.19) completes the proof. 
For ease of computation we will define the point process $\tilde{\eta}_{p, \lambda}^{\omega}$,

$$
\begin{aligned}
\int_{\mathbb{R} \times \mathbb{R}^{d}} f(E, x) d \tilde{\eta}_{p, \lambda}^{\omega}(E, x) & =\sum_{j} f\left(\beta_{L}\left(E_{j}-\lambda\right), \frac{p l_{L}}{L}\right), \\
& =\operatorname{Tr}\left(f\left(\beta_{L}\left(H_{B_{p}(L)}^{\omega}-\lambda\right), \frac{p l_{L}}{L}\right)\right)
\end{aligned}
$$

where $\left\{E_{j}\right\}_{j}$ are eigenvalues of $H_{B_{p}(L)}^{\omega}$ (following notation of (1.8) we can write $\left.d \tilde{\eta}_{p, \lambda}^{\omega}(E, x)=\sum_{j} \delta\left(\beta_{L}\left(E_{j}-\lambda\right)-E\right) d E \delta\left(x-\frac{p l_{L}}{L}\right) d x\right)$. One can prove

$$
\mathbb{E}^{\omega}\left[\left|\int f d \xi_{L, \lambda}^{\omega}-\sum_{p} \int f d \tilde{\eta}_{p, \lambda}^{\omega}\right|\right] \longrightarrow 0 \text { as } L \rightarrow \infty, \quad f \in C_{c}\left(\mathbb{R} \times \mathbb{R}^{d}\right)
$$

by observing that, for $f(E, x)=h(E) g(x)$ where $g \in C_{c}\left(\mathbb{R}^{d}\right)$ and $h$ is given by (2.14), we have

$$
\begin{aligned}
& \left|\int f d \xi_{L, \lambda}^{\omega}-\sum_{p} \int f d \tilde{\eta}_{p, \lambda}^{\omega}\right| \leq\left|\int f d \xi_{L, \lambda}^{\omega}-\sum_{p} \int f d \eta_{p, \lambda}^{\omega}\right|+\sum_{p}\left|\int f d \eta_{p, \lambda}^{\omega}-\int f d \tilde{\eta}_{p, \lambda}^{\omega}\right| \\
& \leq\left|\int f d \xi_{L, \lambda}^{\omega}-\sum_{p} \int f d \eta_{p, \lambda}^{\omega}\right| \\
& \quad+\sum_{p} \frac{\max _{n \in B_{p}(L)}\left|g\left(\frac{n}{L}\right)-g\left(\frac{p l_{L}}{L}\right)\right|}{\beta_{L}} \sum_{n \in B_{p}(L)}\left|\operatorname{Im} G\left(z_{L} ; n, n\right)-\operatorname{Im}^{B_{p}}\left(z_{L} ; n, n\right)\right|
\end{aligned}
$$

Repeating the steps of proposition 2.3 , and using density of function of type $f$ in $C_{c}\left(\mathbb{R} \times \mathbb{R}^{d}\right)$ we have (2.28). So using $\tilde{\eta}_{p, \lambda}^{\omega}$ one can note that any limit point of $\xi_{L, \lambda}^{\omega}$ is a limit point of the point process define by:

$$
\eta_{L, \lambda}^{\omega}:=\sum_{p} \tilde{\eta}_{p, \lambda}^{\omega}
$$

Remark 2.4. For bounded interval $I \subset \mathbb{R}$ and bounded set $Q \subset \mathbb{R}^{d}$, using the equation (2.28) on $\chi_{I}(E) \chi_{Q}(x)$ we have

$$
\mathbb{E}\left(\left|\xi_{L, \lambda}^{\omega}(I \times Q)-\eta_{L, \lambda}^{\omega}(I \times Q)\right|\right) \rightarrow 0 \text { as } L \rightarrow \infty
$$


Lemma 2.5. The weak convergence of $\left\{\xi_{L, \lambda}^{\omega}\right\}$ and $\left\{\eta_{L, \lambda}^{\omega}\right\}$ are equivalent, i.e

$$
\lim _{L \rightarrow \infty} \mathbb{E}^{\omega}\left[\left|e^{-\int f d \xi_{L, \lambda}^{\omega}}-e^{-\int f d \eta_{L, \lambda}^{\omega}}\right|\right]=0, \quad \forall f \in C_{c}^{+}\left(\mathbb{R} \times \mathbb{R}^{d}\right) .
$$

Proof. We have $\left|e^{-x}-e^{-y}\right|<|x-y|$ for $x, y>0$; then using this together with (2.28) will give (2.32). Hence the lemma.

Lemma 2.6. Given $D_{\nu}^{\alpha}(\lambda)>0$ and a symmetric bounded interval $I \subset \mathbb{R}$, there exists a sequence $\left\{L_{n}\right\}_{n}$ such that

$$
\lim _{n \rightarrow \infty} L_{n}^{d} \nu\left(\lambda+\beta_{L_{n}}^{-1} I\right)=|I|^{\alpha} D_{\nu}^{\alpha}(\lambda)
$$

Proof. We have

$$
0<D_{\nu}^{\alpha}(\lambda)=\varlimsup_{\epsilon \rightarrow 0} \frac{\nu(\lambda-\epsilon, \lambda+\epsilon)}{(2 \epsilon)^{\alpha}}<\infty .
$$

Choose $\beta_{L+1}^{-1}<\epsilon \leq \beta_{L}^{-1}$, then for interval $I=[-c, c]$ (for $c>0$ ) we have

$$
\lambda+\epsilon I \subseteq \lambda+\beta_{L}^{-1} I \Rightarrow \nu(\lambda+\epsilon I) \leq \nu\left(\lambda+\beta_{L}^{-1} I\right) .
$$

Using $\beta_{L+1}^{\alpha} \epsilon^{\alpha} \geq 1$

$$
\begin{aligned}
\frac{\beta_{L}^{\alpha} \nu\left(\lambda+\beta_{L}^{-1} I\right)}{|I|^{\alpha}} & \geq\left(\frac{\beta_{L}}{\beta_{L+1}}\right)^{\alpha} \frac{\nu(\lambda+\epsilon I)}{(\epsilon|I|)^{\alpha}} \\
& =\left(\frac{\beta_{L}}{\beta_{L+1}}\right)^{\alpha} \frac{\nu(\lambda-c \epsilon, \lambda+c \epsilon)}{(\epsilon|I|)^{\alpha}}
\end{aligned}
$$

From above we get

$$
\begin{aligned}
\sup _{L \geq M} \frac{\beta_{L}^{\alpha} \nu\left(\lambda+\beta_{L}^{-1} I\right)}{|I|^{\alpha}} & \geq\left(\frac{1}{1+\frac{1}{M}}\right)^{d} \sup _{\epsilon \in\left(\beta_{L+1}^{-1}, \beta_{L}^{-1}\right], L \geq M} \frac{\nu(\lambda+\epsilon I)}{(\epsilon|I|)^{\alpha}} \\
& \geq\left(\frac{1}{1+\frac{1}{M}}\right)^{d} \sup _{\epsilon \in\left(0, \beta_{M}^{-1}\right]} \frac{\nu(\lambda+\epsilon I)}{(\epsilon|I|)^{\alpha}} .
\end{aligned}
$$

Here we used the fact that

$$
\bigcup_{L \geq M}\left(\beta_{L+1}^{-1}, \beta_{L}^{-1}\right]=\left(0, \beta_{M}^{-1}\right] \text { and }\left(\frac{\beta_{L}}{\beta_{L+1}}\right)^{\alpha} \geq\left(\frac{1}{1+\frac{1}{M}}\right)^{d} \text {, for } L \geq M \text {. }
$$


Taking limit $M \rightarrow \infty$ in (2.35) and using definition of limsup, we get

$$
\varlimsup_{L \rightarrow \infty} \frac{\beta_{L}^{\alpha} \nu\left(\lambda+\beta_{L}^{-1} I\right)}{|I|^{\alpha}} \geq D_{\nu}^{\alpha}(\lambda) .
$$

Similarly starting with $\epsilon \in\left(\beta_{L+1}^{-1}, \beta_{L}^{-1}\right]$ we get the inequality

$$
\frac{\beta_{L+1}^{\alpha} \nu\left(E+\beta_{L+1}^{-1} I\right)}{|I|^{\alpha}} \leq\left(\frac{\beta_{L+1}}{\beta_{L}}\right)^{\alpha} \frac{\nu(\lambda+\epsilon I)}{(\epsilon|I|)^{\alpha}}
$$

and proceed as in the above argument, with upper bounds now, to get

$$
\varlimsup_{L \rightarrow \infty} \frac{\beta_{L}^{\alpha} \nu\left(E+\beta_{L}^{-1} I\right)}{|I|^{\alpha}} \leq D_{\nu}^{\alpha}(\lambda) .
$$

Putting the inequalities (2.36) and (2.37) we get

$$
\varlimsup_{L \rightarrow \infty} \frac{\beta_{L}^{\alpha} \nu\left(\lambda+\beta_{L}^{-1} I\right)}{|I|^{\alpha}}=D_{\lambda}^{\alpha}(\lambda) .
$$

Now using the fact $\beta_{L}=L^{d / \alpha}$ we have

$$
\varlimsup_{L \rightarrow \infty} L^{d} \nu\left(\lambda+\beta_{L}^{-1} I\right)=|I|^{\alpha} D_{\nu}^{\alpha}(\lambda) .
$$

The above imply that there exist a subsequence $\left\{L_{n}\right\}$ such that

$$
\lim _{n \rightarrow \infty} L_{n}^{d} \nu\left(\lambda+\beta_{L_{n}}^{-1} I\right)=|I|^{\alpha} D_{\nu}^{\alpha}(\lambda) .
$$

\section{$3 \quad$ Proof of the Theorem 1.1.}

We have

$$
\mathbb{E}\left|e^{i t \xi_{L, \lambda}^{\omega}(I \times Q)}-e^{i t \eta_{L, \lambda}^{\omega}(I \times Q)}\right| \leq|t| \mathbb{E}\left(\left|\xi_{L, \lambda}^{\omega}(I \times Q)-\eta_{L, \lambda}^{\omega}(I \times Q)\right|\right)
$$

We are using the following fact

$$
\left|e^{i t x}-e^{i t y}\right|^{2}=2(1-\cos t(x-y))=4 \sin ^{2} \frac{t(x-y)}{2} \leq|t(x-y)|^{2} .
$$


From (2.30) and (2.5) we have

$$
\begin{aligned}
\mathbb{E}\left[e^{i t \eta_{L, \lambda}^{\omega}(I \times Q)}\right] & =\mathbb{E}\left[e^{i t \sum_{p \in \Gamma_{L}} \tilde{\eta}_{p, \lambda}^{\omega}(I \times Q)}\right] \\
& =\mathbb{E}\left[e^{i t \tilde{\eta}_{p, \lambda}^{\omega}(I \times Q)}\right]^{\left|\Gamma_{L}\right|} .
\end{aligned}
$$

By definition of $\tilde{\eta}_{p, \lambda}^{\omega}$ we have

$$
\begin{aligned}
\mathbb{E}\left[e^{i t \tilde{\eta}_{p, \lambda}^{\omega}(I \times Q)}\right] & =\sum_{k=0}^{\infty} e^{i t m} \mathbb{P}\left(\tilde{\eta}_{p, \lambda}^{\omega}(I \times Q)=k\right) \\
& =1+\mathbb{E}\left[\tilde{\eta}_{p, \lambda}^{\omega}(I \times Q)\right]\left(e^{i t}-1\right)+R_{L} .
\end{aligned}
$$

where $R_{L}$ is given by

$$
\begin{aligned}
R_{L}= & \sum_{k=0}^{\infty} e^{i t k} \mathbb{P}\left(\tilde{\eta}_{p, \lambda}^{\omega}(I \times Q)=k\right)-1-\mathbb{E}\left[\tilde{\eta}_{p, \lambda}^{\omega}(I \times Q)\right]\left(e^{i t}-1\right) \\
= & \sum_{k=0}^{\infty} e^{i t k} \mathbb{P}\left(\tilde{\eta}_{p, \lambda}^{\omega}(I \times Q)=k\right)-\sum_{k=0}^{\infty} \mathbb{P}\left(\tilde{\eta}_{p, \lambda}^{\omega}(I \times Q)=k\right) \\
& \quad-\left(e^{i t}-1\right) \sum_{k=0}^{\infty} k \mathbb{P}\left(\tilde{\eta}_{p, \lambda}^{\omega}(I \times Q)=k\right) \\
= & \sum_{k=2}^{\infty}\left(e^{i t k}-k e^{i t}+k-1\right) \mathbb{P}\left(\tilde{\eta}_{p, \lambda}^{\omega}(I \times Q)=k\right) .
\end{aligned}
$$

Set $I_{L, \lambda}=\lambda+\beta_{L}^{-1} I$ and using $\left|e^{i t k}-k e^{i t}+k-1\right| \leq 2 k$ for $k \geq 2$ we get

$$
\begin{aligned}
\left|R_{L}\right| & \leq \sum_{k=2}^{\infty}\left|e^{i t k}-k e^{i t}+k-1\right| \mathbb{P}\left(\tilde{\eta}_{p, \lambda}^{\omega}(I \times Q)=k\right) \\
& =2 \sum_{k=2}^{\infty} k \mathbb{P}\left(\tilde{\eta}_{p, \lambda}^{\omega}(I \times Q)=k\right) \\
& \leq 2 \sum_{k=2}^{\infty} k(k-1) \mathbb{P}\left(\tilde{\eta}_{p, \lambda}^{\omega}(I \times Q)=k\right) \\
& =2 \mathbb{E}\left[\left(\tilde{\eta}_{p, \lambda}^{\omega}(I \times Q)\left(\tilde{\eta}_{p, \lambda}^{\omega}(I \times Q)-1\right)\right]\right. \\
& \leq 2 \mathbb{E}\left[\left(\tilde{\eta}_{p, \lambda}^{\omega}\left(I \times \mathbb{R}^{d}\right)\left(\tilde{\eta}_{p, \lambda}^{\omega}\left(I \times \mathbb{R}^{d}\right)-1\right)\right]\right.
\end{aligned}
$$




$$
\begin{aligned}
& =2 \mathbb{E}\left[\operatorname{Tr}\left(E_{H_{B_{p}(L)}^{\omega}}\left(I_{L, \lambda}\right)\right)\left(\operatorname{Tr}\left(E_{H_{B_{p}(L)}^{\omega}}\left(I_{L, \lambda}\right)\right)-1\right)\right] \\
& \leq 2\left(Q_{\mu}\left(\left|I_{L, \lambda}\right|\right)\left|B_{p}(L)\right|\right)^{2} \quad(\text { using 2.10) } \\
& \leq 2\left(\left|I_{L, \lambda}\right|^{\alpha} l_{L}^{d}\right)^{2} \\
& =O\left(L^{-2 d} l_{L}^{2 d}\right)
\end{aligned}
$$

Using $\left|\Gamma_{L}\right| \simeq O\left(\left(\frac{L}{l_{L}}\right)^{d}\right)$ (see (2.6) $)$ in above we get

$$
\left|\Gamma_{L}\right|\left|R_{L}\right| \leq O\left(\frac{l_{L}^{d}}{L^{d}}\right) \rightarrow 0 \text { as } L \rightarrow \infty .
$$

Combining above with (3.3), (3.2) and (3.1) will give

$$
\begin{aligned}
\lim _{L \rightarrow \infty} \mathbb{E}\left[e^{i t \xi_{L, \lambda}^{\omega}(I \times Q)}\right] & =\lim _{L \rightarrow \infty} \mathbb{E}\left[e^{i t \eta_{L, \lambda}^{\omega}(I \times Q)}\right] \\
& =\lim _{L \rightarrow \infty}\left(1+\frac{\left|\Gamma_{L}\right|\left[\mathbb{E}\left(\tilde{\eta}_{p, \lambda}^{\omega}(I \times Q)\right)\left(e^{i t}-1\right)+R_{L}\right]}{\left|\Gamma_{L}\right|}\right)^{\left|\Gamma_{L}\right|} \\
& =\lim _{L \rightarrow \infty}\left(1+\frac{\left|\Gamma_{L}\right| \mathbb{E}\left(\tilde{\eta}_{p, \lambda}^{\omega}(I \times Q)\right)\left(e^{i t}-1\right)}{\left|\Gamma_{L}\right|}\right)^{\left|\Gamma_{L}\right|} .
\end{aligned}
$$

To compute the limit, we use the subsequence so that (2.33) holds. Using that subsequence we get:

$$
\begin{aligned}
\lim _{n \rightarrow \infty}\left|\Gamma_{L_{n}}\right| \mathbb{E}\left(\tilde{\eta}_{p, \lambda}^{\omega}(I \times Q)\right) & =\lim _{n \rightarrow \infty} \sum_{p \in \Gamma_{L_{n}}} \mathbb{E}\left(\tilde{\eta}_{p, \lambda}^{\omega}(I \times Q)\right) \\
& =\lim _{n \rightarrow \infty} \mathbb{E}\left(\eta_{L_{n}, \lambda}^{\omega}(I \times Q) \quad \text { (using (2.30) }\right) \\
& =\lim _{n \rightarrow \infty} \mathbb{E}\left(\xi_{L_{n}, \lambda}^{\omega}(I \times Q) \quad(\text { using 2.31) }\right. \\
& =\lim _{n \rightarrow \infty} \sum_{n \in L_{n} Q} \mathbb{E}\left(\left\langle\delta_{n}, E_{H^{\omega}}\left(\lambda+\beta_{L_{n}}^{-1} I\right) \delta_{n}\right\rangle\right) \\
& =|Q| \lim _{L_{n} \rightarrow \infty} L_{n}^{d} \nu\left(\lambda+\beta_{L_{n}}^{-1} I\right) \\
& =|I|^{\alpha} D_{\nu}^{\alpha}(\lambda)|Q| \quad(\text { using(2.33) })
\end{aligned}
$$

Using above in the (3.7) together with the fact $\left(1+\frac{z_{n}}{n}\right)^{n} \rightarrow e^{z}$, whenever $z_{n} \rightarrow z$ as $n \rightarrow \infty$ gives

$$
\mathbb{E}\left[e^{i t \xi_{L_{n}, \lambda}^{\omega}(I \times Q)}\right] \stackrel{n \rightarrow \infty}{\longrightarrow} e^{|I|^{\alpha} D_{\nu}^{\alpha}(\lambda)|Q|\left(e^{i t}-1\right)} .
$$


Which shows that $\left\{\xi_{L_{n}, \lambda}^{\omega}(I \times Q)\right\}$ converges in distribution to a Poisson random variable with parameter $|I|^{\alpha} D_{\nu}^{\alpha}(\lambda)|Q|$.

Acknowledgement: We thank M Krishna for useful discussion and valuable comments. We also thank the referees for their helpful comments and suggestions.

\section{References}

[1] Applebaum, David: Lévy processes and stochastic calculus, Cambridge Studies in Advances Mathematics 116, second edition, Cambridge: Cambridge University Press, 2009.

[2] Aizenman, Michael; Molchanov, Stanislav: Localization at large disorder and at extreme energies: an elementary derivation, Commun. Math. Phys. 157(2) (1993), 245-278.

[3] Aizenman, Michael; Warzel, Simone: The Canopy Graph and Level Statistics for Random Operators on Trees, Mathematical Physics, Analysis and Geometry, 9(4) (2006), 291-333.

[4] Carmona, René; Lacroix, Jean: Spectral theory of random Schrodinger operators, Boston, Birkhauser, 1990.

[5] Carmona, René; Klein, Abel; Martinelli, Fabio: Anderson localization for Bernoulli and other singular potentials, Comm. Math. Phys. 108(1) (1987), 41-66.

[6] Combes, Jean-Michel; Hislop, Peter D; Klopp, Frédéric: An optimal Wegner estimate and its application to the global continuity of the integrated density of states for random Schrödinger operators, Duke Math. J. 140(3) (2007), 469-498.

[7] Combes, Jean-Michel; Germinet, François; Klein, Abel: Generalized Eigenvalue-Counting Estimates for the Anderson Model, J Stat Physics 135(2) (2009), 201-216.

[8] Daley, D.J; Vere-Jones: An Introduction to the Theory of Point Processes II, General theory and structure, Springer, New York, 2008. 
[9] Demuth, Michael; Krishna, M: Determining Spectra in Quantum Theory, Progress in Mathematical Physics. 44, Birkhäuser, Boston, 2004.

[10] Dolai, Dhriti; Krishna, M: Level Repulsion for a class of decaying random potentials, Markov Processes and Related Fields (to appear), arXiv:1305.5619[math.SP].

[11] Dolai, Dhriti; Krishna, M: Poisson statistics for Anderson model with singular randomness, Journal of the Ramanujan Mathematical Society(to appear), arXiv:1408.4251[math.SP].

[12] Geisinger, Leander: Poisson eigenvalue statistics for random Schrödinger operators on regular graphs, Annales Henri Poincaré, Springer Basel, 2014. 1-28.

[13] Germinet, François; Klopp, Frédéric: Spectral statistics for the discrete Anderson model in the localized regime, Spectra of random operators and related topics, (2011), 11-24, RIMS Kôkyûroku Bessatsu, B27, Res. Inst. Math. Sci. (RIMS), Kyoto.

[14] Hislop, Peter D; Krishna. M: Eigenvalue statistics for random Shroödinger operators with non-rank one perturbation, arXiv:1409.2328 math-ph].

[15] H. L. Cycon; R. G. Froese; W. Kirsch; B. Simon: Schrödinger operators, with application to quantum mechanics and global geometry, Texts and Monographs in Physics, Springer-Verlag, Berlin, 1987.

[16] Kallenberg, Olav: Lectures on Random Measures, Institute of Statistics Mimeo Series No. 963, 1974.

[17] Kallenberg, Olav: Foundations of Modern Probability, Springer, New York 2002.

[18] Kaminaga, M.; Krishna, M.; Nakamura, S: A note on the analyticity of density of states, Journal of Statistical Physics, 149(3), (2013), 496-504.

[19] Killip, Rowan; Nakano, Fumihiko: Eigenfunction Statistics in the Localized Anderson Model, Ann. Henri Poincare 8(1) (2007), 27-36. 
[20] Kirsch, Werner: An Invitation to Random Schrödinger Operators (With an appendix by Frédéric Klopp) Panor. Synthèses, 25, Random Schrödinger operators, 1 1-119, Soc. Math. France, Paris, 2008.

[21] Kotani, S; Nakano, Fumihiko: Level statistics of one-dimensional Schrödinger operators with random decaying potential, Preprint, 2012.

[22] Krishna, M: Continuity of intregrated density of states-independent randomness, Proc. Ind. Acad. Sci. 117(3) (2007), 401-410.

[23] Minami, Nariyuki: Local Fluctuation of the Spectrum of a Multidimensional Anderson Tight Binding Model, Commun. Math. Phys. 177(3) (1996), 709-725.

[24] Molchanov, S. A. The local structure of spectrum of a random onedimensional Schrödinger operator, Trudy Sem. Petrovsk, 8 (1982), 195210 .

[25] Nakano, Fumihiko: The repulsion between localization centers in the Anderson model, J. Stat. Phys. 123 (2006), 803-810.

[26] Nakano, Fumihiko: Infinite divisibility of random measures associated to some random Schrödinger operators, Osaka J. Math, 46 (2009), 845862 .

[27] Nakano, Fumihiko: Distribution of localization centers in some discrete random systems. Reviews in Mathematical Physics 19.09 (2007): 941965.

[28] Reed, Michael; Simon, Barry: Method of modern mathematical physics I Functional Analysis, Academic Press, 1978.

[29] Rogers, C, A : Hausdorff Measures, Cambridge University Press, 1970.

[30] Stollmann, Peter: From uncertainty principles to Wegner estimates, Math. Phys. Anal. Geom. 13, (2010), 145-157.

[31] Tautenhahn, M; Veselić, I: Minami's estimate: Beyond rank one perturbation and monotonicity, Ann. Henri Poincaré 15 (2014), 737-754. 